\title{
Sympathetic and parasympathetic neurons are likely to be absent in the human vestibular and geniculate ganglia: an immunohistochemi- cal study using elderly cadaveric specimens
}

\author{
By \\ Masato YAMAUCHI ${ }^{1}$, Masahito YAMAMOTO ${ }^{1}$, Kei KITAMURA ${ }^{\mathbf{1}}$, \\ Masaaki KASAHARA ${ }^{1}$, Satoru MATSUNAGA ${ }^{1}$, \\ Gen MURAKAMI ${ }^{2}$, Shin-ichi ABE ${ }^{1}$. \\ ${ }^{1}$ Department of Anatomy, Tokyo Dental College, 2-9-18 Misaki-cho, Chiyoda-ku, Tokyo 101-0061, Japan \\ ${ }^{2}$ Division of Internal Medicine, Iwamizawa Asuka Hospital, 297 Shibun cho, Iwamizawa 068-0833, Japan \\ -Received for Publication, February 17, 2016- \\ Key Words: Vestibular and geniculate ganglia, neuronal nitric oxide, tyrosine hydroxylase, facial nerve, immunohistochemistry,
human anatomy \\ Summary: The vestibular and geniculate ganglia of the ear in experimental animals carry both of the tyrosine hydroxylase \\ (TH)-positive sympathetic neurons and the neuronal nitric oxide synthase (nNOS)-positive parasympathetic neurons. With \\ an aid of immunohistochemistry, we examined these ganglia as well as the horizontal part of the facial nerve using specimens \\ from 10 formalin-fixed elderly cadavers. The submandibular ganglion from the same cadavers was used for the positive \\ control for both markers. Although there was a nonspecific reaction in nuclei for the present antibody of nNOS, these ganglia \\ were unlikely to contain either nNOS- or TH-positive neurons. However, we did not deny a possibility that the absence was \\ a result of degeneration with aging. In contrast, the facial nerve horizontal part consistently contained both of TH-positive- \\ and nNOS-positive fibers. These fibers might regulate blood supply to the facial nerve and the dysregulation leads to edema \\ to elevate pressure on the nerve within its osseous canal.
}

\section{Introduction}

In the human head, parasympathetic ganglion cells for secretory motor or vasomotor innervation, those are usually positive for neuronal nitric oxide synthase or nNOS, are located in the ciliary, pterygopalatine, otic and submandibular ganglia' ${ }^{1}$. However, all of these parasympathetic ganglia also contain sympathetic ganglion cells those are positive for tyrosine hydroxylase or $\mathrm{TH}^{2}$. In contrast to the autonomic motor nerves, the geniculate ganglion of the facial nerve as well as the vestibular ganglion (Scarpa's ganglion) of the vestibulocochlear nerve has been known as a purely sensory ganglion ${ }^{1}$. However, in the vestibular ganglion of experimental animals, many research groups identified an expression of $\mathrm{nNOS}^{3-7)}$ or $\mathrm{TH}^{8)}$. Likewise, in experimental animals, the geniculate ganglion contains $\mathrm{nNOS}^{3}, 4,9$ ). Consequently, with an aid of immunohistochemistry, we conducted the present study to examine the existence of sympathetic and/or parasympathetic nerve elements in the human geniculate and vestibular ganglia.

\section{Materials and Methods}

The study was performed in accordance with the provisions of the Declaration of Helsinki 1995 (as revised in Edinburgh 2000). We examined 10 temporal bones from donated male cadavers with ages of 71-87 years (mean, 82 years). The cause of death had been ischemic heart failure. These cadavers had been donated to Tokyo Dental College for research and education on human anatomy, and their use for research had been approved by the university ethics committee. The donated cadavers had been fixed by arterial perfusion of $10 \% \mathrm{v} / \mathrm{v}$ formalin solution and stored in $50 \% \mathrm{v} / \mathrm{v}$ ethanol solution for more 


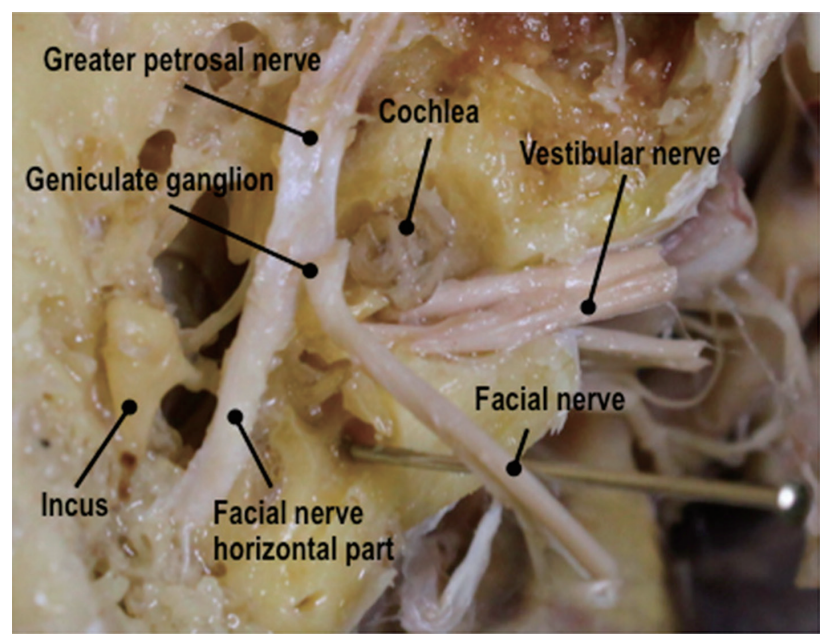

Fig. 1. Dissection of the vestibular and geniculate ganglia in the skull base. An 82-year-old man. Left side. To move the proximal part of the facial nerve posteriorly, a pin is inserted into the semicircular canal. The cochlea was broken during the dissection.

than 3 months. During minute dissection under surgical microscope (Fig. 1), we prepared 4 small tissue blocks from each of the bones: 1) the vestibular nerves at the bottom of the internal auditory meatus, 2) the geniculate ganglion of the facial nerve, 3 ) the horizontal part of the facial nerve distal to the geniculate ganglion. Using histological sections (an equipment for postgraduate education in the Department of Otorhinolaryngology), we learnt the topographical anatomy of the vestibular ganglion (Fig. 2): the aforementioned specimen No. 1 was most likely to contain the vestibular ganglion although the ganglion cell cluster extends long along the nerve ${ }^{10)}$. In addition, for the positive control, the submandibular ganglion was obtained in the same cadavers at a routine surgical approach from the submandibular triangle.

After performing routine procedures for paraffin-embedded histology, we prepared 20-30 serial sections to find the best site of these ganglia. Most sections were stained with hematoxylin and eosin (HE) and some were used for immunohistochemistry. According to Hinata et al. ${ }^{11)}$, the primary antibodies used for nerve immunohistochemistry were 1) rabbit polyclonal anti-human nNOS (1:100; Cell Signaling Technology, Beverly, MA) and 2) rabbit polyclonal anti-human TH (1:100; Millipore-Chemicon ab152, Temecula, CA). The secondary antibody (incubation for $30 \mathrm{~min}$; dilution 1:1000; Histofine Simple Stain Max-PO, Nichirei, Tokyo) was labeled with horseradish peroxidase (HRP), and antigen-antibody reactions were detected by the HRP-catalyzed reaction with diaminobenzidine (incubation for 3-5 min; Histofine Simple Stain DAB, Nichirei, Tokyo). All samples were counterstained with hematoxylin. Nega-

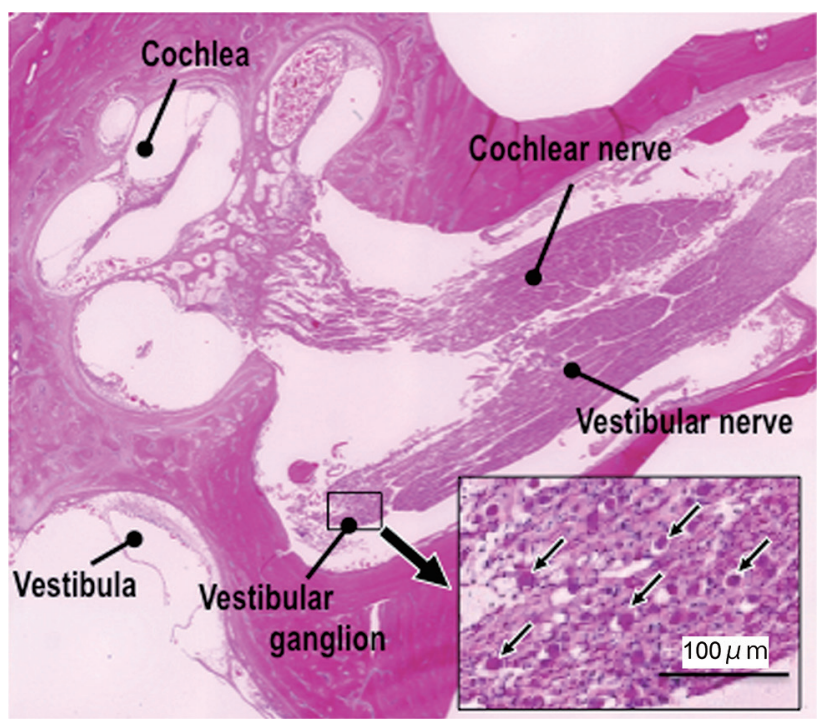

Fig. 2. Topographical anatomy of the vestibular ganglion. An 84-yearold man. HE staining. An insert displays ganglion cells at a site indicated by an indicating bar with "vestibular ganglion".

tive controls consisted of samples without the primary antibody.

\section{Results}

The submandibular ganglion as the positive control always contained both nNOS-positive cells and TH-positive cells: the positive cytoplasm colored deep brown. The maximum diameter of neurons ranged from 30-40 micron. Nuclei of all ganglion cells were, much or less, appeared to display nNOS immunoreactivity for the present antibody. Thus, nuclei of the TH-positive cells appeared to be positive also for nNOS (Fig. 3). However, we did not consider this result as a double-positive.

From all 10 cadavers, we successfully obtained a ganglion cell cluster of the vestibular ganglion that was composed of 20-40 ganglion cells per section (20-30 micron at the maximum diameter). Likewise, the geniculate ganglion contained 5-10 ganglion cells per section (20-40 micron at the maximum diameter). Nuclei of all of these neurons were strongly positive for nNOS, but the cytoplasm also appeared to be weakly positive (Fig. 4). In contrast, in both ganglia, TH-reactivity was not seen not only in the nucleus but the cytoplasm. Moreover, no nNOS-positive fibers were seen in and around these ganglion cell clusters. In contrast, the horizontal part of the facial nerve contained both nNOS- and TH-positive nerve fibers although a proportion in the sectional area (area/area) was roughly estimated as less than 5\% (Fig. $4 \mathrm{CF}$ ). In addition, the vestibular nerve in the proximal 

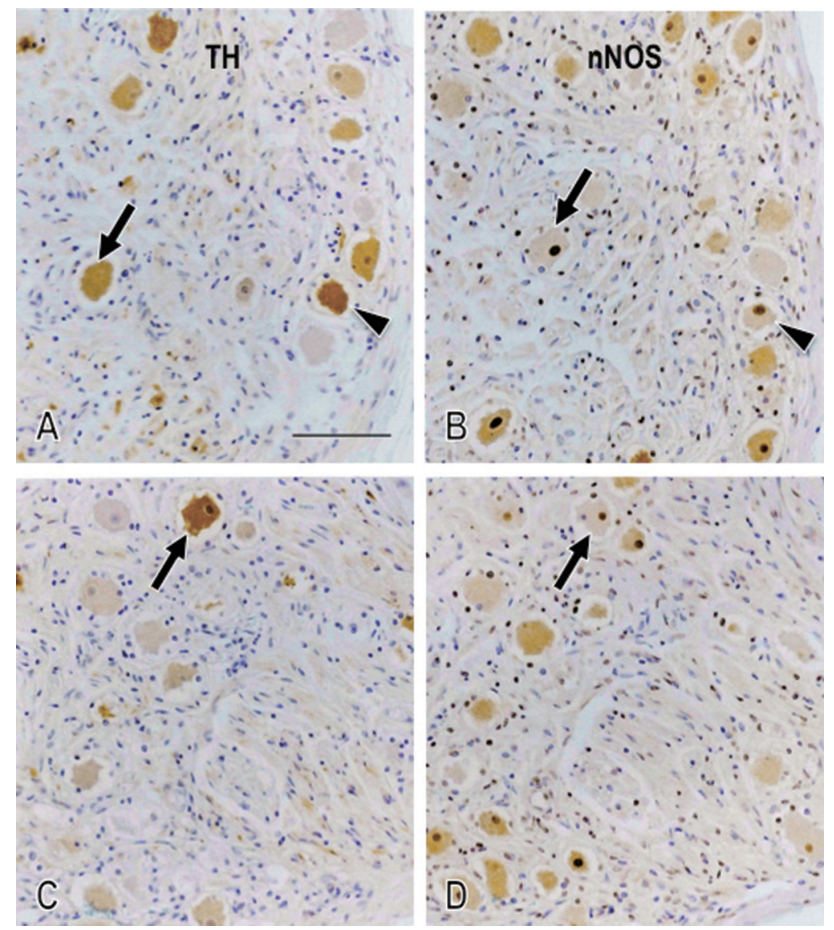

Fig. 3. Immunohistochemistry of the submandibular ganglion: a positive control. An 85-year-old man. Left side. Panels A and B (or $\mathrm{C}$ and $\mathrm{D}$ ) exhibit serial sections. Panels $\mathrm{A}$ and $\mathrm{C}$ display immunohistochemistry of tyrosine hydroxylase (TH), while panels B and $\mathrm{D}$ that of neuronal nitric oxide synthase (nNOS). In panels $\mathrm{A}$ and $\mathrm{B}$ (or C and D), arrows or arrowheads indicate the same ganglion cells. Nuclei of the TH-positive cells appeared to be also positive for nNOS. Scale bar in panel A, $0.1 \mathrm{~mm}$.

side of the ganglion contained few TH-positive fibers (figure, not shown).

\section{Discussion}

Although a successful staining of nNOS immunohistochemistry is obtained in less than half of formalin-fixed cadaveric specimens after long preservation in ethanol solution ${ }^{12)}$, the present observations revealed absence of either nNOS-positive or TH-positive neurons in the human vestibular and geniculate ganglia. However, we did not deny a possibility that this absence was a result of degeneration with aging. Positive immunoreactivity of the nucleus seemed to be non-specific because of the expression seen in all ganglion cells including those in the submandibular ganglion. In the latter ganglion, TH-positive cells sometimes exhibited nNOS positivity of the nucleus. To our knowledge, a double expression of the TH and nNOS was not reported in the head. Actually, a member of sympathetic nerve markers, neuropeptide $\mathrm{Y}$, is likely to coexist nNOS in the vestibular ganglion
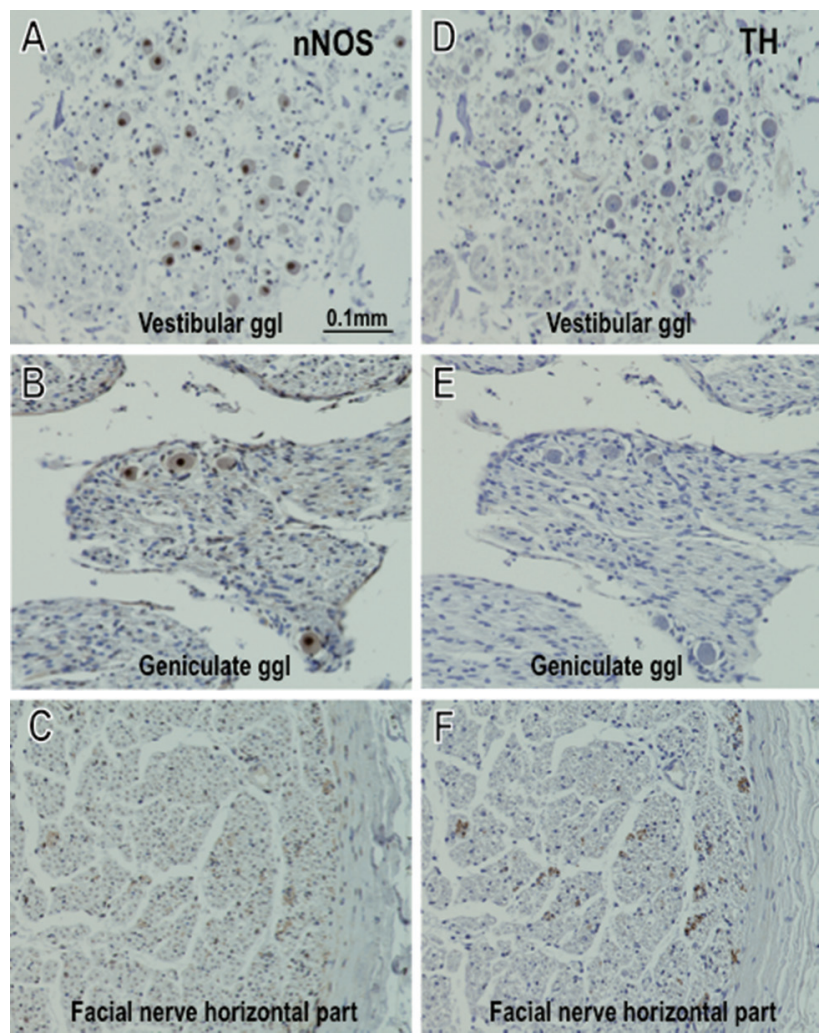

Fig. 4. Immunohistochemistry of the vestibular and geniculate ganglia. An 80-year-old man. Panels A-C (or D-F) display immunohistochemistry of neuronal nitric oxide synthase or nNOS (tyrosine hydroxylase or TH). Panels A and D (or B and E, C and F) exhibit serial sections. Abundant nNOS-positive nuclei are seen in these ganglia (arrowheads in panels A and B), but TH-positive neurons appeared to be absent (panels D and E). The facial nerve horizontal part contains both nNOS- and TH-positive nerve fibers (arrows in panels $\mathrm{C}$ and $\mathrm{F}$ ).

cells of 4-month old female pigs ${ }^{7}$. However, in humans, such a paradoxical expression between the sympathetic and parasympathetic markers may be limited to occur in some enteric neurons: Anlauf et al. ${ }^{13}$ ) described a group of neurons those are double positive for a cholinergic marker and TH. In addition, choline acetyltransferase is likely to co-exist in the sensory nerve fibers in the human vestibular periphery ${ }^{14}$.

Based on immunohistochemical data from the experimental animals, Michel et al. ${ }^{9)}$ postulated a critical contribution of nitric oxide to regulate blood supply to the facial nerve. According to them, nitric oxide regulates vascular tone of nutrient blood vessels in the perineural sheath and endoneurium and the dysregulation leads to edema and elevated pressure on the nerve within its osseous canal. Actually, we demonstrated nNOS-positive nerve fibers, possibly coming from the otic ganglion, on the facial nerve horizontal part. However, in the other paper $^{3,4)}$, his research group emphasized that the vestib- 
ular ganglion does not show clear nNOS immunoreactivity, while soluble guanylyl cyclase is present in the cytoplasm. In the present study, the facial nerve also contained TH-positive fibers. Yamashita et al. ${ }^{8)}$ considered that these nerves may influence on capillary permeability. Overall, the vestibular and geniculate ganglia seemed to be purely sensory in the human elderly.

\section{Acknowledgment}

We are grateful to people who donated their bodies after their death to Tokyo Dental College for research and education on human anatomy without any economic benefit. We also thank to their families for agreement of the donation as well as patience for waiting the return their bones after study.

\section{Reference}

1) Williams PL: Gray's Anatomy. 38th ed., Churchill Livingstone, Edinburgh, 1995; 1227-1251.

2) Kiyokawa H, Katori Y, Cho KH, Murakami G, Kawase T, Cho $\mathrm{BH}$ : Reconsideration of the autonomic cranial ganglia: an immunohistochemical study of mid-term human fetuses. Anat Rec 2012; 295:141-149.

3) Hess A, Bloch W, Arnhold S, Andressen C, Stennert E, Addicks K, Michel O: Nitric oxide synthase in the vestibulochochlear system of mice. Brain Res 1998; 813:97-102.

4) Hess A, Bloch W, Su J, Stennert E, Addicks K, Michel O: Expression of inducible nitric oxide-synthase in the vestibular system of hydropic guinea pigs. Neurosci Lett 1999; 264:145-148.
5) Takumida M, Anniko M: Localization of nitric oxide synthase idoforms (NOSI, II, and III) in the vestibular end organs of the guinea pig. ORL J Otorhinolaryngol Relat Spec 1998; 60:67-72.

6) Takumida M, Anniko M: Detection of nitric oxide in the guinea pig inner ear, using a combination of aldehyde fixative and 4.5-diaminofluorescein diacetate. Acta Otolaryngol 2001; 121: 460-464.

7) Dudek A, Sienkiewicz W, Kaleczyc J: Immunohistochemical characterization of neurons in the vestibular ganglion (Scarpa's ganglion) of the pig. Pol J VetSci 2012; 15:499-507.

8) Yamashita H, Bagger-Sjöbäck D, Sekitani T: Distribution of tyrosine hydroxylase-like immunofluorescence in guinea pig vestibular ganglia and sensory areas. Auris Nasus Larynx 1992; 19:6368.

9) Michel O, Hess A, Krolzig M, Stennert E, Addick J, Bloch W: Involvement of nitric synthase in the physiology and pathophysiology of facial nerve function and dysfunction. Eur Arch Otorhinolaryngol 2000; 257:188-192.

10) Sato H, Sando I, Takahashi H: Three-dimensional anatomy of human Scarpa's ganglion. Laryngoscope 1992; 102:1056-1063.

11) Hinata N, Hieda K, Sasaki H, Murakami G, Abe S, Matsubara A, Miyake H, Fujisawa M: Topohistology of sympathetic and parasympathetic nerve fibers in branches of the pelvic plexus: an immunohistochemical study using donated elderly cadavers. Anat Cell Biol 2014; 47:55-65.

12) Ishiyama G, Hinata $N$, Kinugasa $Y$, Hieda $K$, Murakami G, Fujimiya F: Nerves to the internal anal sphincter: an immunohistochemical study using donated elderly cadavers. Surg Radiol Anat 2014; 36:1033-1042.

13) Anlauf M, Schåfer MKH, Eiden L, Weihe E: Chemical coding of the human gastrointestinal nervous system: cholinergic, VIPergic, and catecholaminergic phenotypes. J Comp Neurol 2003; 459:90111.

14) Popper P, Ishiyama A, Lopez I, Wackym PA: Calcitonin gene-related peptide and choline acetyltransferase colocalization in the human vestibular periphery. Audiol Neurootol 2002; 7:298-302. 